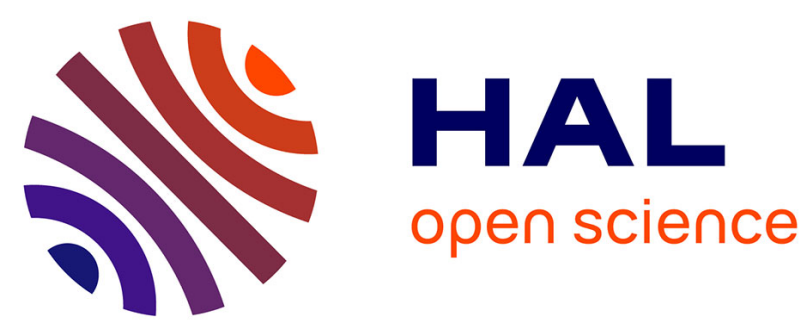

\title{
Characteristic X-ray radiation and Auger electrons from resonant coherently excited highly charged ions under channeling
}

\author{
V.V. Balashov, A. Sokolik, A. Stysin
}

\section{To cite this version:}

V.V. Balashov, A. Sokolik, A. Stysin. Characteristic X-ray radiation and Auger electrons from resonant coherently excited highly charged ions under channeling. The Seventh International Symposium on Swift Heavy Ions in Matter, Jun 2008, Lyon, France. hal-00255895

\section{HAL Id: hal-00255895 \\ https://hal.science/hal-00255895}

Submitted on 4 Aug 2008

HAL is a multi-disciplinary open access archive for the deposit and dissemination of scientific research documents, whether they are published or not. The documents may come from teaching and research institutions in France or abroad, or from public or private research centers.
L'archive ouverte pluridisciplinaire HAL, est destinée au dépôt et à la diffusion de documents scientifiques de niveau recherche, publiés ou non, émanant des établissements d'enseignement et de recherche français ou étrangers, des laboratoires publics ou privés. 


\title{
Characteristic X-ray radiation and Auger electrons from resonant coherently excited highly charged ions under channeling
}

\author{
V.V. Balashov ${ }^{a}$ A.A. Sokolik ${ }^{b}$ A.V. Stysin ${ }^{a}$ \\ ${ }^{a}$ Institute of Nuclear Physics, Moscow State University, 119991 Moscow, Russia \\ ${ }^{\mathrm{b}}$ Institute of Spectroscopy, Russian Academy of Sciences, 142190 Troitsk, Moscow \\ Region, Russia
}

\begin{abstract}
The density-matrix approach to treat the resonant coherent excitation of swift ions in oriented crystals is applied for a unified theoretical description of the charge state distribution of relativistic resonant coherently excited ions, their characteristic Xray radiation and, as a new aspect, the Auger electron production from doubly excited states.
\end{abstract}

Key words: highly charged ions, channeling, resonant coherent excitation, Auger electrons

PACS: $61.85 .+\mathrm{p}, 32.60 .+\mathrm{i}, 32.80 . Z \mathrm{~b}, 32.90 .+\mathrm{a}$.

\section{Introduction}

In our previous works $[1,2]$ we have successfully applied the density matrix approach [3] to treat the resonant coherent excitation (RCE) [4] of planar- 
channeled relativistic ions. The present work is stimulated by recent RCE experiments of the Tokyo RCE collaboration including their unambiguous observation of the azimuthal anisotropy of the characteristic X-ray radiation from planar-channeled ions [5], double RCE experiments [6], and trajectory dependent measurements of the charge state fraction distribution of the ion beam $[7]$.

In Sect. 2 we suggest to use experimental technique of [7] to study not only the charge state distribution but also the characteristic X-rays from $\mathrm{RCE}$ planar channeled ions resolved over the transverse entrance coordinate $z_{\text {in }}$ of the incoming ion. Our treatment shows the close connection of the angular distribution of the X-rays with the to geometrical properties of the in-crystal electric field.

Sect. 3 is devoted to a new direction in physics of autoionization phenomena opened by experiment [6]. Our preliminary results reported in [2] point to the considerable yield of the Auger electrons and their high angular anisotropy to be observed in double RCE experiments with helium-like ions. We develop further this description by taking into account the Stark mixing between the "ladder-type" excited $2 \mathrm{p}^{2}:{ }^{1} \mathrm{D}_{2}$ state and the nearly lying autoionizing state 2s2p : ${ }^{1} \mathrm{P}_{1}$ and show its influence on the RCE observables in the autoionization region.

\section{Angular anisotropy of characteristic X-ray radiation}

Recently we showed in detail [8], how certain geometrical properties of the in-crystal electric field manifest themselves in the angular anisotropy of the 
characteristic X-ray radiation from resonant coherently excited ions. Usually, following Komaki et al. [9], planar-channeling RCE is treated in the ion rest frame $(x, y, z)$ which $x$ axis is directed along the beam and $z$ axis is perpendicular to the channel. One of the results of the paper [8] is that RCE on a single $(k, l)$ resonance can be most conveniently treated in a special coordinate system, where its $X$ axis is determined by the Lorentz transformation of the corresponding harmonic of the field into its both scalar $\varphi\left(\vec{r}^{\prime}, t^{\prime}\right)$ and vector $\vec{A}\left(\vec{r}^{\prime}, t^{\prime}\right)$ components in the ion rest frame while the $Z$ axis remains perpendicular to the channeling plane. To show the main idea of [8] in this short presentation, we neglect here all effects of the vector potential induced in the ion rest frame and, using the dipole approximation for the electric field in this frame, take a projection of its vector $\vec{E}$ on the channeling plane for the direction of axis $X$. One can easily turn from the aforementioned coordinate system $(x, y, z)$ to $(X, Y, Z)$ one by rotation on some angle $\theta^{\prime}=\arctan \left[(\vec{E})_{y} /(\vec{E})_{x}\right]$ in the channeling plane.

Considering the geometrical properties of the resonant part of the in-crystal electric field in the ion rest frame, we show that it is a superposition of two oscillating fields, which act in $X$ and $Z$ directions and have $c_{X}$ and $c_{Z}$ amplitudes with a $\pi / 2$ phase shift between them. Dipole excitation $1 \mathrm{~s} \rightarrow 2 \mathrm{p}$ by these fields occurs into state $|\psi\rangle=c_{X}\left|2 \mathrm{p}_{X}\right\rangle+c_{Z}\left|2 p_{Z}\right\rangle$, where $\left|2 \mathrm{p}_{j}\right\rangle$ is the $2 \mathrm{p}$ electron state with zero projection onto the $j$-th axis. The angular distribution of E1 photons emitted in the decay of this state has the form

$$
W \propto\left\{\left|c_{Z}\right|^{2} n_{X}^{2}+\left(\left|c_{X}\right|^{2}+\left|c_{Z}\right|^{2}\right) n_{Y}^{2}+\left|c_{X}\right|^{2} n_{Z}^{2}\right\}
$$

where $n_{j}$ is the projection onto the $j$ axis of the unit vector guiding an observation direction. This distribution can be viewed as a superposition of two 
$\sin ^{2} \theta_{\gamma}$ dipole distributions of photons coming from the decay of $2 \mathrm{p}_{X}$ and $2 \mathrm{p}_{Z}$ states, with a vanishing interference term. This implies that $W_{Y}=W_{X}+W_{Z}$, where $W_{j}$ is the differential photon yield in the $j$ axis direction. Thus the angular distribution of X-rays takes extremal values in the directions of axes of the $(X, Y, Z)$ frame, taking intermediate values in other directions. This simple picture illustrates the advantage of using the new frame to consider the properties of the RCE X-ray radiation under planar channeling.

Motivated by the recent experiments with trajectory resolved RCE observables [7] and with X-ray anisotropy measurements [5], we have performed calculations of the trajectory resolved anisotropy of X-rays from $423 \mathrm{MeV} / \mathrm{u}$ $\mathrm{Fe}^{24+}$ ions in the $(2 \overline{2} 0)$ Si channel, coherently excited at the $(k, l)=(2,-1)$ resonance. In order to enable convenient trajectory resolving, we take the target thickness $0.9 \mu \mathrm{m}$ corresponding to one-quarter of the period of the ion transverse oscillations in the channel. Trajectory resolved angular distributions $U\left(\theta_{\gamma}, \varphi_{\gamma}\right)$ of X-ray photons are calculated via the integration of (1) along the corresponding ion trajectories. The results of our calculations at $\theta=2.57^{\circ}$ are presented in Fig. 1 as a function of the impact parameter $z_{\text {in }}$ of the ion in the units of the half-width $d / 2$ of the channel. It is seen that $U_{Y}=U_{X}+U_{Z}$ and, for the most part of photons, $U_{Y} \approx 2 U_{Z}$.

This calculation directly relates to recent trajectory non-resolved RCE measurements [5] with $423 \mathrm{MeV} / \mathrm{u} \mathrm{Fe}^{24+}$ ions at the $(k, l)=(2,-1)$ resonance in a $21 \mu \mathrm{m}$-thick target which show that the photon yield in the $y$-axis direction is approximately twice larger than that in the $z$-axis direction. In this case the angle $\theta^{\prime}$ is small and therefore the photon yields along corresponding directions of the $(X, Y, Z)$ and $(x, y, z)$ frames differ insignificantly: $U_{Y} \approx U_{y}$, $U_{Z} \approx U_{z}$. That is why the experimentally measured ratio $U_{y} / U_{z}$ is close to 
our aforementioned ratio $U_{Y} / U_{Z} \approx 2$. Larger differences can arise in RCE situations with larger $\theta^{\prime}$ values (see [2] for the presentation of these calculations in more detail).

\section{Double resonant coherent excitation}

The "Ladder type" double resonant coherent excitation of autoionizing states of helium-like $\operatorname{Ar}^{16+}$ ions passing through the $(2 \overline{2} 0)$ planar channel of a $27 \mu \mathrm{m}$ thick Si crystal was investigated for the first time experimentally by the Tokyo group [6]. The initial $1 \mathrm{~s}^{2}:{ }^{1} \mathrm{~S}_{0} \rightarrow 1 \mathrm{~s} 2 \mathrm{p}:{ }^{1} \mathrm{P}_{1}$ step of excitation occurs under action of the crystal field harmonic with $(k, l)=(1,-2)$. The consequent excitation to the doubly excited state $2 \mathrm{p}^{2}:{ }^{1} \mathrm{D}_{2}$ is due to the $(k, l)=(1,1)$ harmonic. Resonant conditions for both steps are fulfilled simultaneously for ions with energy $387.90 \mathrm{MeV} / \mathrm{u}$ entering the crystal at $\theta=1.23^{\circ}$.

Developing our earlier study [2] on the double resonant coherent excitation, we show in Fig. 2 the considerable Stark mixing between $2 \mathrm{p}^{2}:{ }^{1} \mathrm{D}_{2}$ and $2 \mathrm{~s} 2 \mathrm{p}:{ }^{1} \mathrm{P}_{1}$ autoionizing states closely lying in the excitation spectrum of the $\mathrm{Ar}^{16+}$ ion. Taken into account, this effect leads to an improved agreement between our theoretical calculations and the survival fraction experimental data shown in Fig. 3 normalized as in [6] relative to the background level. On the same way we estimate the total yield of Auger electrons emitted in the process under consideration (Fig. 4). As Fig. 4 shows, about $40 \%$ of the total probability of the electron loss by the propagating ion at the resonance maximum is due to the Auger decay of both $2 \mathrm{p}^{2}:{ }^{1} \mathrm{D}_{2}$ and $2 \mathrm{~s} 2 \mathrm{p}:{ }^{1} \mathrm{P}_{1}$ autoionizing states. We used [10-14] for the required data on radiative and Auger decay lifetimes of excited states of the highly charged ions, on excitation and ionization cross sections 
in their collisions with the in-channel electrons.

The calculations show that the doubly excited states produced in the resonant coherent excitation process are aligned and, as a result, that the angular distributions of X-rays and Auger electrons from their decay are strongly anisotropic. They are given in the ion rest frame in Fig. 5. The characteristic features of the angular distribution of the Auger electrons are due to the dominant contribution of the decay of the aligned ${ }^{1} \mathrm{D}_{2}$ state. Note also that its interference with the ${ }^{1} \mathrm{P}_{1}$ component violates pure reflection symmetries of the decay pattern.

\section{Conclusion}

Presented results demonstrate the extending scope of applications of the density matrix approach in theoretical RCE studies, and can serve to guide new experiments.

We have found, that the ionic excited state alignment in the RCE process and the characteristic features of the accompanying X-ray radiation can be considered especially clear in a coordinate frame taken according to the geometrical properties of the resonant part of the in-crystal electric field. Systematic experimental studies of RCE X-ray radiation, including trajectory resolved measurements, can play an important role to reveal the main regularities of the ions behavior in the crystal environment under channeling conditions.

Our results concerning the autoionizing state production in double RCE show large yield of Auger electrons at the resonance maximum and the essential anisotropy of their angular distribution. Taking into account the Stark mixing 
between different autionization states brings our results into better agreement with experiment. Future double-RCE experiments on Auger electrons production and special measurements of their angular distribution, in combination with those on the yield and the angular anisotropy of the X-ray photons from the autoionizing and intermediate ion states, can be an important contribution to correlated experimental and theoretical studies on the boundary between physics of autoionization phenomena and channeling.

\section{Acknowledgements}

We are thankful to Prof. T. Azuma and his group at the Tokyo Metropolitan University for useful discussions. The work was supported by the Russian Foundation for Basic Research (grants 06-02-16367, 08-02-09213). A.A.S. acknowledges the support from the Dynasty Foundation and the Russian Science Support Foundation. 


\section{References}

[1] V.V. Balashov, A.A. Sokolik, Optika i Spectroskopiya 103 (2007) 785 [Optics and Spectroscopy 103 (2007) 761].

[2] V.V. Balashov, A.A. Sokolik, A.V. Stysin, Zh. Exp. Teor. Phys. 134 (2008) 1 [JETP 107 (2008) 133].

[3] V.V. Balashov, I.V. Bodrenko, Moscow Univ. Phys. Bull. No. 1 (2001) 27; Phys. Lett. A 352 (2006) 129; Nucl. Instr. Meth. Phys. Res. B 245 (2006) 52.

[4] V.V. Okorokov, Yad. Phys. 70 (2007) 1213 [Phys. At. Nucl. 70 (2007) 1174].

[5] T. Azuma, Y. Takabayashi, C. Kondo, T.Muranaka, K.Komaki, Y.Yamazaki, E.Takada, T.Murakami, Phys. Rev. Lett. 97 (2006) 145502.

[6] Y. Nakano, S. Masugi, T. Muranaka, T.Azuma, C.Kondo, A.Hatakeyama, K.Komaki, Y.Yamazaki, E.Takada, T.Murakami, J. Phys.: Conf. Ser. 58 (2007) 395.

[7] C. Kondo, S. Masugi, T. Muranaka, A.Ishikawa, Y.Nakano, T.Azuma, A.Hatakeyama, K.Komaki, Y.Nakai, Y.Yamazaki, E.Takada, T.Murakami, Nucl. Instr. Meth. Phys. Res. B 256 (2007) 157.

[8] V.V. Balashov, A.A. Sokolik, A.V. Stysin, to be published.

[9] K. Komaki, T. Azuma, T. Ito, Y. Takabayashi, Y. Yamazaki, M. Sano, M. Torikoshi, A. Kitagawa, E. Takada, T. Murakami, Nucl. Instr. Meth. Phys. Res. B 146 (1998) 19.

[10] Yu. Ralchenko, A.E. Kramida, J. Reader, and NIST ASD Team (2008). NIST Atomic Spectra Database (version 3.1.5), [Online]. Available: http://physics.nist.gov/asd3 [2008, May 27].

[11] L.A. Vainshtein and U.I. Safronova, At. Dat. Nucl. Dat. Tables 21 (1978) 49. 
[12] C. Lin, W.R. Johnson, A. Dalgarno, Phys. Rev. A 15 (1977) 154.

[13] A.D. Parks, D.H. Sampson, Astrophys. J. 178 (1972) 571.

[14] W.A. Bernshtam, Yu.V. Ralchenko, Y. Maron, J. Phys. B: At. Mol. Opt. Phys. $33(2000) 5025$. 


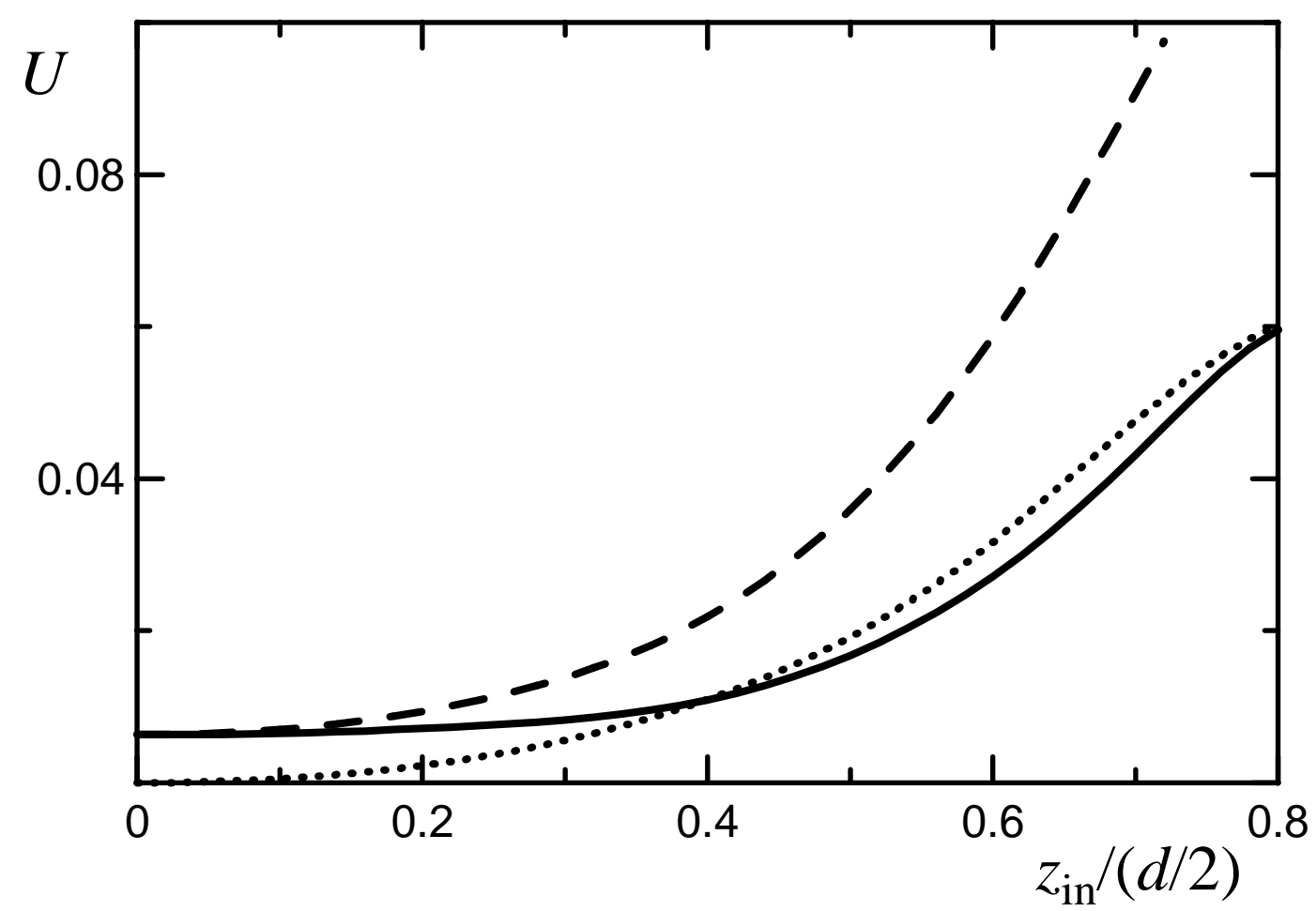

Fig. 1. Trajectory resolved differential yield of X-ray radiation from $423 \mathrm{MeV} / \mathrm{u}$ $\mathrm{Fe}^{24+}$ ions under RCE conditions at $(k, l)=(2,-1)$ resonance in the directions of $X$ axis (solid line), $Y$ axis (dashed line) and $Z$ axis (dotted line). 

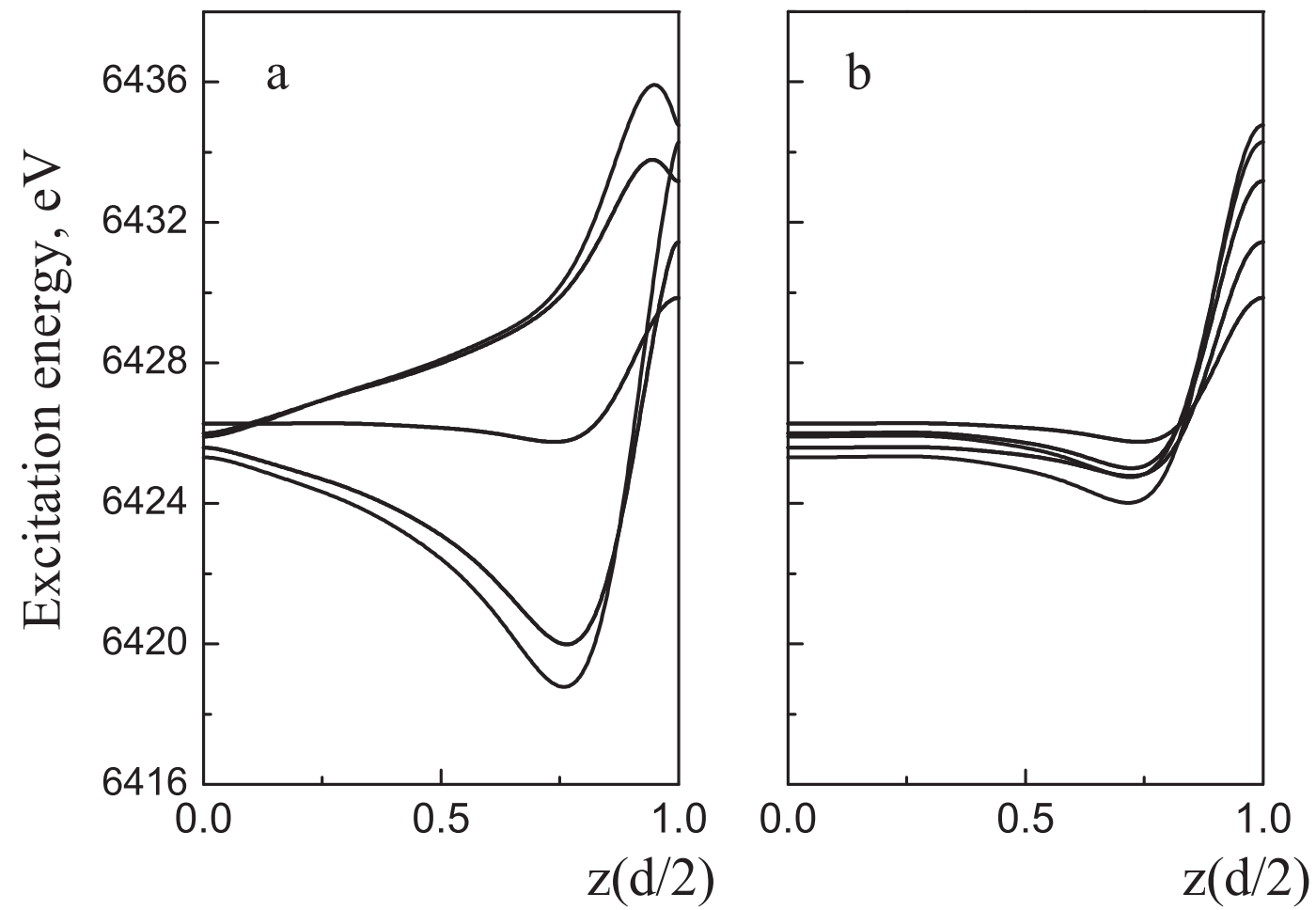

Fig. 2. Calculated energy levels of doubly excited heliumlike $\operatorname{Ar}^{16+}$ ion in the $(2 \overline{2} 0)$ planar channel of a Si crystal with Stark mixing of $2 \mathrm{~s} 2 \mathrm{p}:{ }^{1} \mathrm{P}_{1}$ and $2 \mathrm{p}^{2}:{ }^{1} \mathrm{D}_{2}$ states (a) taken into account and (b) not taken into account. 


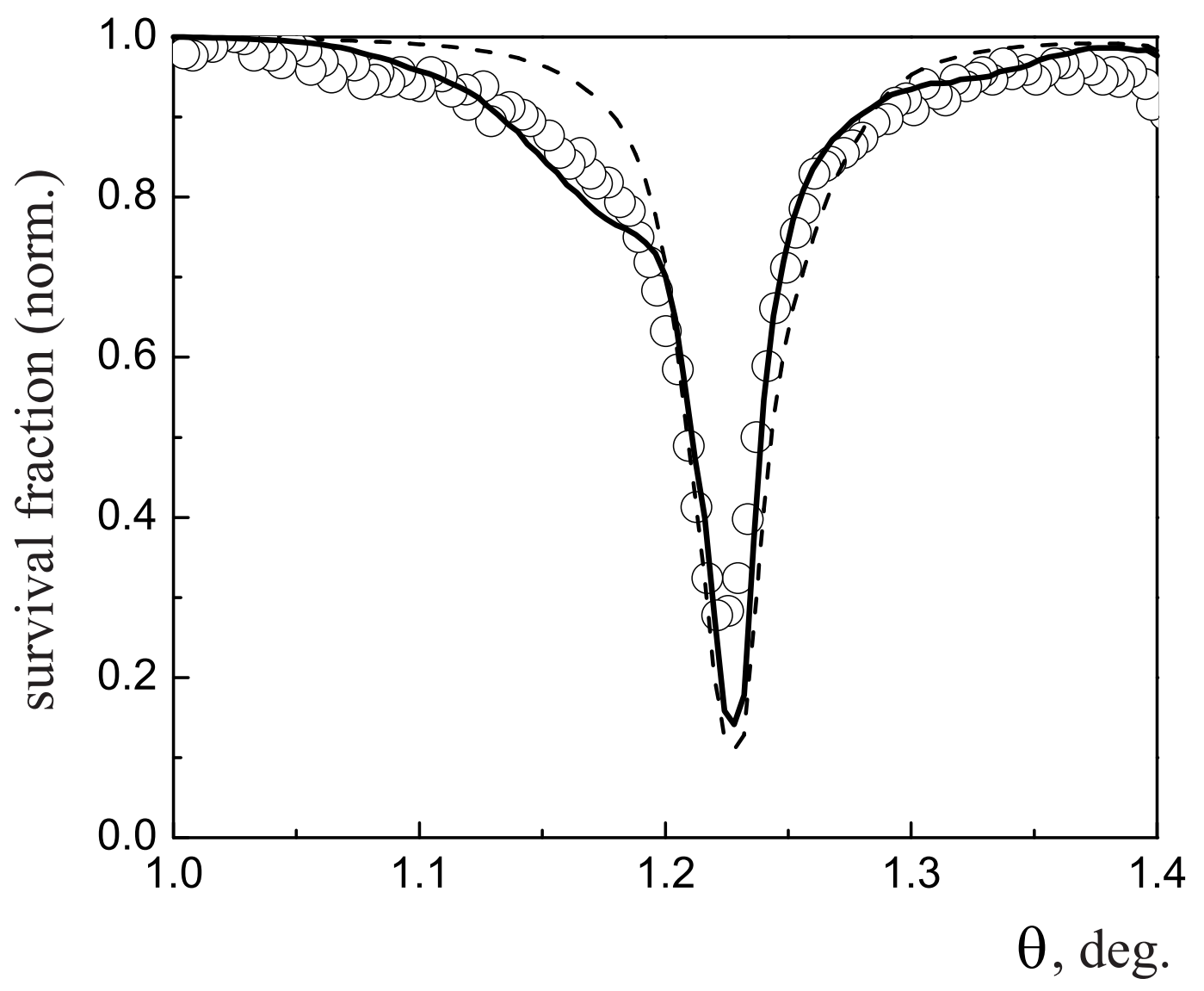

Fig. 3. Normalized survival fraction of $387.90 \mathrm{MeV} / \mathrm{u} \mathrm{Ar}^{16+}$ ions passing through a $27 \mu \mathrm{m}$ Si crystal. Solid line - calculations with $2 \mathrm{p}^{2}:{ }^{1} \mathrm{D}_{2}-2 \mathrm{~s} 2 \mathrm{p}:{ }^{1} \mathrm{P}_{1}$ mixing, dash line - calculations without $2 \mathrm{~s} 2 \mathrm{p}:{ }^{1} \mathrm{P}_{1}$ state. Experimental data (circles) are taken from $[6]$. 


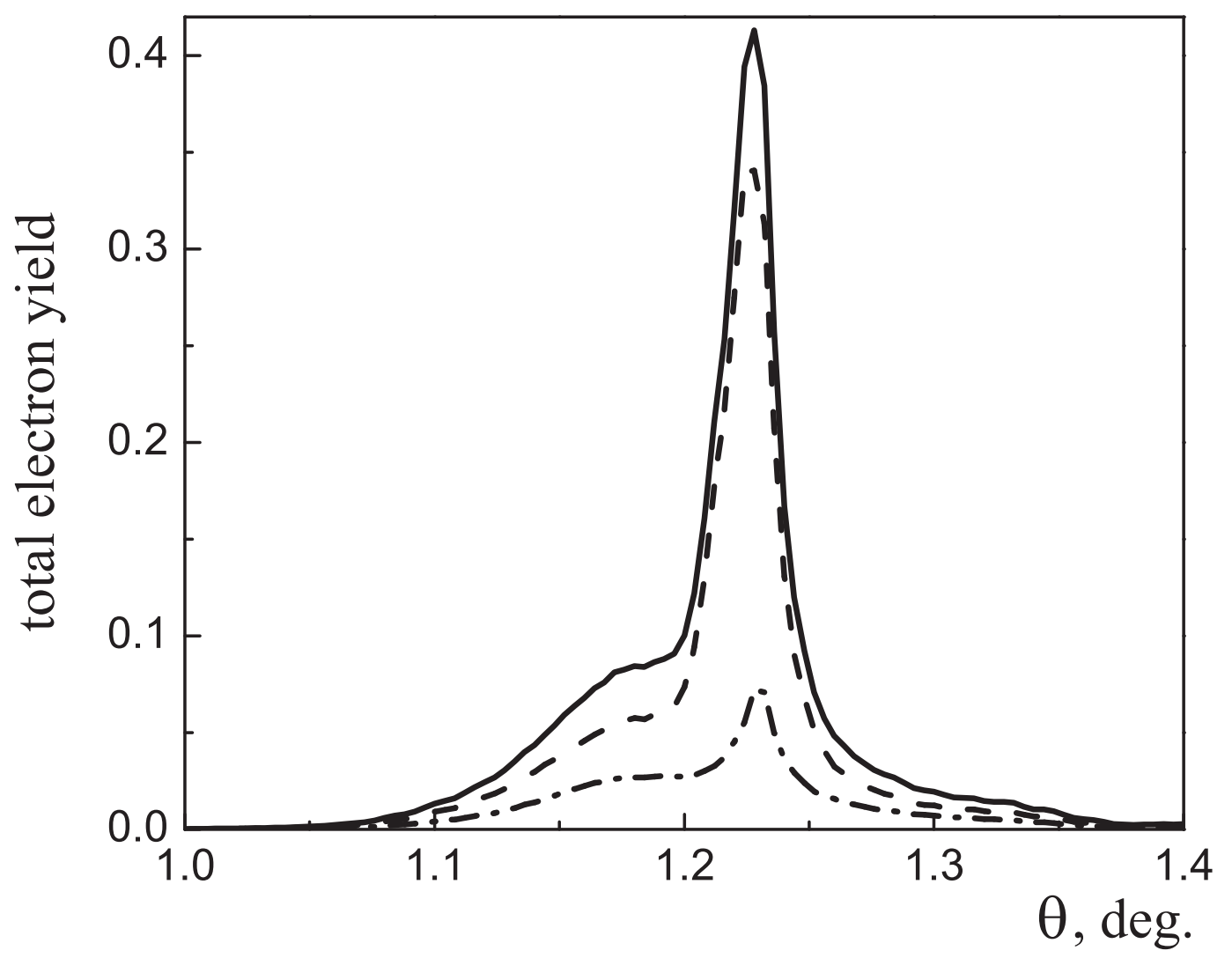

Fig. 4. Total Auger electron yield for $387.90 \mathrm{MeV} / \mathrm{u} \mathrm{Ar}^{16+}$ ions passing through a $27 \mu \mathrm{m}$ Si crystal (solid line). Dash line and dash-dot lines - contributions from $2 \mathrm{p}^{2}:{ }^{1} \mathrm{D}_{2}$ and $2 \mathrm{~s} 2 \mathrm{p}:{ }^{1} \mathrm{P}_{1}$ respectively. 

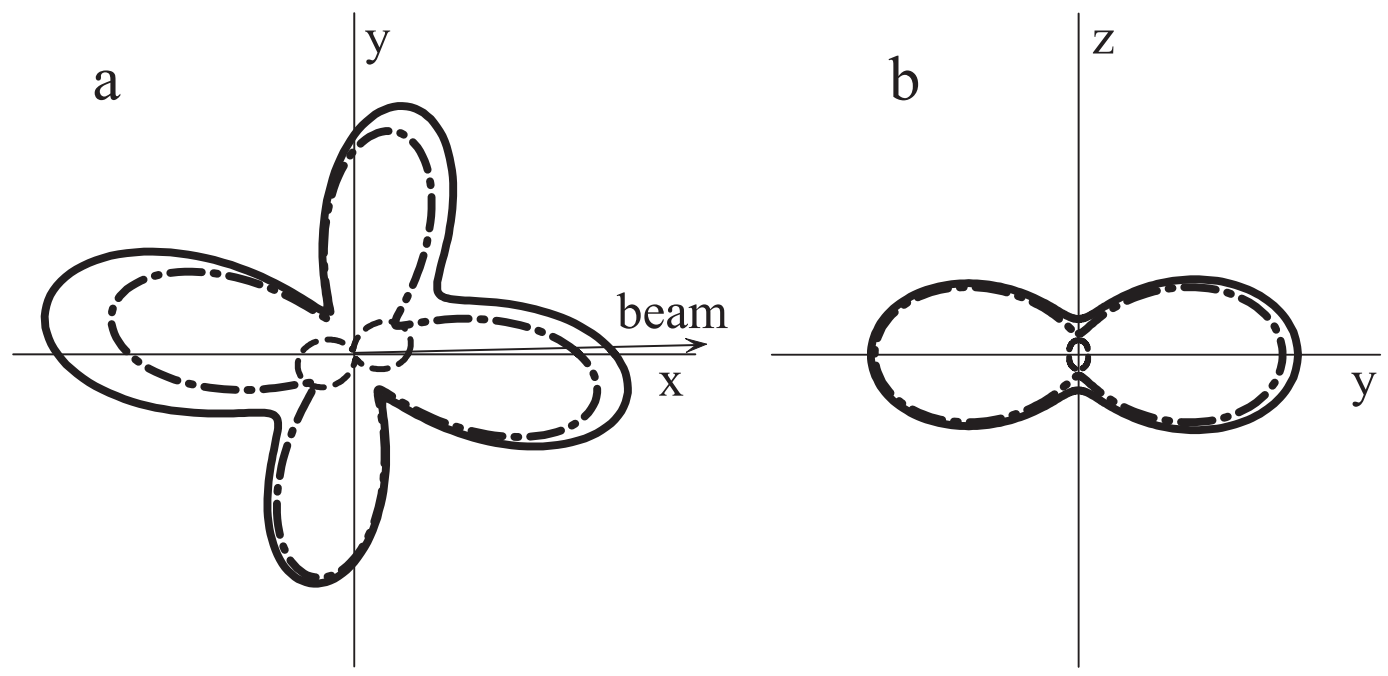

Fig. 5. Calculated angular distribution of Auger electrons: (a) in the channel plane, (b) perpendicular to the beam. Dash lines - contribution from $2 \mathrm{~s} 2 \mathrm{p}:{ }^{1} \mathrm{P}_{1}$, dash-dot lines — contribution from $2 \mathrm{p}^{2}:{ }^{1} \mathrm{D}_{2}$. Solid line — sum including the interference term. 